\title{
Necessary and Sufficient Conditions for Stable Conjugate Duality*
}

\author{
R. S. Burachik $\uparrow^{\dagger}$ V. Jeyakumar $\ddagger$ and Z-Y. $\mathrm{Wu}^{\S}$ \\ PREPRINT VERSION
}

\begin{abstract}
The conjugate duality, which states that inf $x \in X \quad \phi(x, 0)=\max _{v \in Y^{\prime}}-\phi^{*}(0, v)$, whenever a regularity condition on $\phi$ is satisfied, is a key result in convex analysis and optimization, where $\phi: X \times Y \rightarrow \mathbb{R} \cup\{+\infty\}$ is a convex function, $X$ and $Y$ are Banach spaces, $Y^{\prime}$ is the continuous dual space of $Y$ and $\phi^{*}$ is the Fenchel-Moreau conjugate of $\phi$. In this paper, we establish a necessary and sufficient condition for the stable conjugate duality,

$$
\inf _{x \in X}\left\{\phi(x, 0)+x^{*}(x)\right\}=\max _{v \in Y^{\prime}}\left\{-\phi^{*}\left(-x^{*}, v\right)\right\}, \forall x^{*} \in X^{\prime},
$$

and obtain a new global dual regularity condition, which is much more general than the popularly known interior-point type conditions, for the conjugate duality. As a consequence we present an epigraph closure condition which is necessary and sufficient for a stable Fenchel-Rockafellar duality theorem. In the case where one of the functions involved in the duality is a polyhedral convex function, we also provide generalized interior-point conditions for the epigraph closure condition. Moreover, we show that a stable Fenchel's duality for sublinear functions holds whenever a subdifferential sum formula for the functions holds. As applications, we give general sufficient conditions for a minimax theorem, a subdifferential composition formula and for duality results of convex programming problems.
\end{abstract}

Keywords: conjugate duality, constraint qualifications, convex programming, polyhedral functions, sublinear functions.

AMS (2000) Subject Classification: 90C25, 49J52, 49J53, 49J35, 65K10.

*The authors are grateful to Professor Wen Song for his comments on the preliminary version of the paper.

†Engenharia de Sistemas e Computacao, COPPE-UFRJ CP 68511, Rio de Janeiro-RJ, CEP 21945-970, Brazil. E-mail: regi@cos.ufrj.br

${ }^{\ddagger}$ School of Mathematics, University of New South Wales, Sydney, NSW 2052, Australia. E-mail: jeya@maths.unsw.edu.au

$\S$ Department of Mathematics and Computer Science, Chongqing Normal University, Chongqing 400047, P. R. China; School of Mathematics, University of New South Wales, Sydney, NSW 2052, Australia. E-mail: zhiyouwu@Maths.unsw.edu.au 


\section{Introduction}

A fundamental duality scheme for studying the convex minimization problem

$$
\min f(x), \quad x \in X,
$$

where $f: X \rightarrow \mathbb{R} \cup\{+\infty\}$, is by a representation function $\phi: X \times Y \rightarrow \mathbb{R} \cup\{+\infty\}$ such that $\phi(x, 0)=f(x)$. In which case the dual problem associated to (1.1) is given by

$$
\max -\phi^{*}(0, v), \quad v \in Y^{\prime},
$$

where $X$ and $Y$ are Banach spaces with the duals $X^{\prime}$ and $Y^{\prime}$ respectively, and $\phi^{*}$ is the Fenchel-Moreau conjugate of $\phi$. A conjugate duality states that

$$
\inf _{x \in X} \phi(x, 0)=\max _{v \in Y^{\prime}}-\phi^{*}(0, v)
$$

whenever a regularity condition on $\phi$ is satisfied. In other words, under the regularity condition, there is no duality gap and the dual problem has a solution. The conjugate duality, which is a key to the study of convex optimization, constrained best approximation and interpolation (see $[7,8,9]$ ), enables, for instance, one to find the optimal solution by solving the corresponding dual optimization problem. A central question in convex analysis and optimization is to find a general regularity condition for the conjugate duality. From the point of view of applications, it is vital to find conditions on $\phi$, which characterize the stable conjugate duality:

$$
\inf _{x \in X}\left\{\phi(x, 0)+x^{*}(x)\right\}=\max _{v \in Y^{\prime}}\left\{-\phi^{*}\left(-x^{*}, v\right)\right\}, \forall x^{*} \in X^{\prime} .
$$

Various (primal) regularity conditions for the duality have been given in the literature (see $[14,20]$ and other reference therein). However, these regularity conditions are either (global) interior-point type conditions $[1,16,18]$ which frequently restrict applications or are based on local conditions. In recent years, it has been shown in $[5,6,4]$ that Fenchel's duality holds under a dual epigraph condition, which is strictly weaker than the usual interior-point conditions $[1,12]$, and the bounded linear regularity condition [2], which have played important roles in convex analysis and optimization.

The purpose of this paper is to establish a necessary and sufficient condition for the stable conjugate duality and derive new global dual regularity conditions, which are much more general than the popularly known interior-point type conditions, for conjugate duality results. We also present an epigraph closure condition that is necessary and sufficient for a stable Fenchel-Rockafellar duality theorem. In the case where one of the functions involved in the duality is a polyhedral convex function, we provide generalized interior-point conditions for the epigraph closure condition. Moreover, we show that a stable Fenchel duality for sublinear functions holds whenever a subdifferential sum formula for the functions holds. As applications, we give general sufficient conditions for a minimax theorem, a subdifferential composition formula and for duality results of convex programming problems involving polyhedral constraints. 


\section{Preliminaries: Epigraphs of Conjugate Functions}

We begin by fixing some definitions and notations. We assume throughout that $X$ and $Y$ are Banach spaces. The continuous dual space of $X$ will be denoted by $X^{\prime}$ and will be endowed with the weak* topology. For the set $D \subset X$ the closure of $D$ will be denoted cl $D$. If a set $A \subset X^{\prime}$, the expression cl $A$ will stand for the weak* closure. The indicator function $\delta_{D}$ is defined as $\delta_{D}(x)=0$ if $x \in D$ and $\delta_{D}(x)=+\infty$ if $x \notin D$. The support function $\sigma_{D}$ is defined by $\sigma_{D}(u)=\sup _{x \in D} u(x)$. The normal cone of $D$ is given by $N_{D}(x):=\left\{v \in X^{\prime}: \sigma_{D}(v)=v(x)\right\}=\left\{v \in X^{\prime}: v(y-x) \leq\right.$ $0, \forall y \in D\}$ when $x \in D$, and $N_{D}(x):=\emptyset$ when $x \notin D$. Let $f: X \rightarrow \mathbb{R} \cup\{+\infty\}$ be a proper lower semi-continuous convex function. Then, the conjugate function of $f, f^{*}: X^{\prime} \rightarrow \mathbb{R} \cup\{+\infty\}$, is defined by

$$
f^{*}(v)=\sup \{v(x)-f(x) \mid x \in \operatorname{dom} f\}
$$

where the domain of $f, \operatorname{dom} f$, is given by $\operatorname{dom} f=\{x \in X \mid f(x)<+\infty\}$. The epigraph of $f$, Epi $f$, is defined by

$$
\text { Epi } f=\{(x, r) \in X \times \mathbb{R} \mid x \in \operatorname{dom} f, f(x) \leq r\} .
$$

The subdifferential of $f, \partial f: X \rightrightarrows X^{\prime}$ is defined as

$$
\partial f(x)=\left\{v \in X^{\prime} \mid f(y) \geq f(x)+v(y-x), \forall y \in X\right\} .
$$

Note also that $\partial \delta_{D}=N_{D}$. If $f: X \rightarrow \mathbb{R} \cup\{+\infty\}$ is a proper lower semi-continuous sublinear function, i.e., $f$ is convex and positively homogeneous $(f(0)=0$, and $f(\lambda x)=\lambda f(x), \forall x \in X, \forall \lambda \in(0, \infty))$, then $\partial f(0)$ is non-empty and for each $x \in$ $\operatorname{dom} f$,

$$
\partial f(x)=\{v \in \partial f(0) \mid v(x)=f(x)\} .
$$

Applying the well-known Moreau-Rockafellar Theorem (see e.g. [18, Theorem $3.2]$ ) we obtain the conclusion in the lemma below.

Lemma 2.1 [18] Let $X$ be a Banach space. Let $f, g: X \rightarrow \mathbb{R} \cup\{+\infty\}$ be proper lower semi-continuous convex functions such that $\operatorname{dom} f \cap \operatorname{dom} g \neq \emptyset$. Then $\operatorname{Epi}(f+g)^{*}=$ $\operatorname{cl}\left(\right.$ Epi $f^{*}+$ Epi $\left.g^{*}\right)$.

If both $f$ and $g$ are proper lower semi-continuous and sublinear functions then it easily follows from Lemma 2.1 that

$$
\partial(f+g)(0)=\operatorname{cl}(\partial f(0)+\partial g(0))
$$

For the details see [20].

\section{Stable Conjugate Duality}

In this section we establish characterizations of the conjugate duality and derive a global dual condition for conjugate duality. We begin by establishing a necessary and sufficient condition for the conjugate duality. 
Proposition 3.1 Let $\phi: X \times Y \rightarrow \mathbb{R} \cup\{+\infty\}$ be a proper and lower semi-continuous convex function. Let $\alpha:=\inf _{x \in X} \phi(x, 0)$ be finite. Then the following statements are equivalent.

(i) $\inf _{x \in X} \phi(x, 0)=\max _{v \in Y^{\prime}}-\phi^{*}(0, v)$.

(ii) $(0,0,-\alpha) \in \operatorname{Epi} \phi^{*}+\left(\{0\} \times Y^{\prime} \times \mathbb{R}_{+}\right)$.

Proof. $[(i) \Longrightarrow(i i)]$ By assumption (i), there exists $v \in Y^{\prime}$ such that $-\phi^{*}(0, v)=$ $\alpha \in \mathbb{R}$. Since $\left(0, v, \phi^{*}(0, v)\right) \in \operatorname{Epi} \phi^{*}$ and $(0,-v, 0) \in\{0\} \times Y^{\prime} \times \mathbb{R}_{+}$we can write

$(0,0,-\alpha)=\left(0,0, \phi^{*}(0, v)\right)=\left(0, v, \phi^{*}(0, v)\right)+(0,-v, 0) \in$ Epi $\phi^{*}+\left(\{0\} \times Y^{\prime} \times \mathbb{R}_{+}\right)$.

$[(i i) \Longrightarrow(i)]$ From (ii), there exist $(u, v, \delta) \in$ Epi $\phi^{*}, w \in Y^{\prime}$ and $\varepsilon \in \mathbb{R}_{+}$such that

$$
(0,0,-\alpha)=(u, v, \delta)+(0, w, \varepsilon)=(u, v+w, \delta+\varepsilon)
$$

Then $u=0, v=-w$ and $\delta+\varepsilon+\alpha=0$. Now, by Fenchel's inequality, for each $x \in X$ and $v^{\prime} \in Y^{\prime}, \phi(x, 0)+\phi^{*}\left(0, v^{\prime}\right) \geq 0$. Using this for $v^{\prime}:=v$ we get

$$
\begin{aligned}
\inf _{x \in X} \phi(x, 0) & \geq-\phi^{*}(0, v) \\
& \geq-\delta \\
& =\varepsilon+\alpha \\
& \geq \alpha=\inf _{x \in X} \phi(x, 0) .
\end{aligned}
$$

Thus, $\inf _{x \in X} \phi(x, 0)=\max _{y^{\prime} \in Y^{\prime}}-\phi^{*}\left(0, y^{\prime}\right)=-\phi^{*}(0, v)$, and hence (i) holds.

We now establish a necessary and sufficient condition for the stable conjugate duality.

Theorem 3.1 (Stable Conjugate Duality) Let $\phi: X \times Y \rightarrow \mathbb{R} \cup\{+\infty\}$ be a proper and lower semi-continuous convex function. Suppose that $\alpha:=\inf _{x \in X}\{\phi(x, 0)\}<$ $+\infty$. Then the following statements are equivalent.

(i) $\inf _{x \in X}\left\{\phi(x, 0)+x^{*}(x)\right\}=\max _{v \in Y^{\prime}}\left\{-\phi^{*}\left(-x^{*}, v\right)\right\}, \forall x^{*} \in X^{\prime}$.

(ii) $\operatorname{Epi~} \phi^{*}+\left(\{0\} \times Y^{\prime} \times \mathbb{R}_{+}\right)$is weak* closed.

Proof. [(i) $\Longrightarrow$ (ii)]. Let $M=\{(x, y) \in X \times Y \mid y=0\}$. Then, Epi $\delta_{M}^{*}=\{0\} \times$ $Y^{\prime} \times \mathbb{R}_{+}$. Since $\alpha=\inf _{x \in X} \phi(x, 0)<+\infty$ we have that $\operatorname{dom} \phi \cap M \neq \emptyset$. So we can apply Lemma 2.1 to write

$$
\operatorname{cl}\left(\operatorname{Epi} \phi^{*}+\{0\} \times \mathrm{Y}^{\prime} \times \mathbb{R}_{+}\right)=\operatorname{cl}\left(\operatorname{Epi} \phi^{*}+\operatorname{Epi} \delta_{M}^{*}\right)=\operatorname{Epi}\left(\phi+\delta_{M}\right)^{*}
$$

Fix now an element $\left(x^{*}, y^{*}, r\right) \in \operatorname{cl}\left(\right.$ Epi $\left.\phi^{*}+\{0\} \times \mathrm{Y}^{\prime} \times \mathbb{R}_{+}\right)$. By the above equality we have that $\left(\phi+\delta_{M}\right)^{*}\left(x^{*}, y^{*}\right) \leq r$. So,

$$
r \geq\left(\phi+\delta_{M}\right)^{*}\left(x^{*}, y^{*}\right) \geq-\inf _{x \in X}\left\{\phi(x, 0)-x^{*}(x)\right\} .
$$

By assumption (i) we get 


$$
\inf _{x \in X}\left\{\phi(x, 0)-x^{*}(x)\right\}=\max _{v \in Y^{\prime}}\left\{-\phi^{*}\left(x^{*}, v\right)\right\} .
$$

Hence,

$$
r \geq\left(\phi+\delta_{M}\right)^{*}\left(x^{*}, y^{*}\right) \geq \phi^{*}\left(x^{*}, v\right),
$$

for some $v \in Y^{\prime}$. This implies that $\left(x^{*}, v, r\right) \in$ Epi $\phi^{*}$. Therefore, $\left(x^{*}, y^{*}, r\right)=$ $\left(x^{*}, v, r\right)+\left(0, y^{*}-v, 0\right) \in \operatorname{Epi} \phi^{*}+\{0\} \times \mathrm{Y}^{\prime} \times \mathbb{R}_{+}$and (ii) holds. [(ii) $\left.\Longrightarrow(\mathrm{i})\right]$. Note that the assumption $\alpha=\inf _{x \in X} \phi(x, 0)<+\infty$ yields $\alpha\left(x^{*}\right):=\inf _{x \in X} \phi(x, 0)+x^{*}(x)<$ $+\infty$ for all $x^{*} \in X^{\prime}$. If $x^{*} \in X^{\prime}$ is such that $\alpha\left(x^{*}\right)=-\infty$, then Fenchel-Young inequality yields the equality in (i) for this choice of $x^{*}$. Now assume that $x^{*} \in X^{\prime}$ is such that $\alpha\left(x^{*}\right)>-\infty$, so our basic assumption yields $\alpha\left(x^{*}\right) \in \mathbb{R}$. We can write

$$
\alpha\left(x^{*}\right)=\inf _{x \in X} \phi(x, 0)+x^{*}(x)=-\left(\phi+\delta_{M}\right)^{*}\left(-x^{*}, 0\right) \in \mathbb{R} .
$$

Note also that $\alpha(0)=\inf _{x \in X} \phi(x, 0)<+\infty$ and hence $M \cap \operatorname{dom} \phi \neq \emptyset$, which gives

$$
\begin{aligned}
\left(-x^{*}, 0,-\alpha\left(x^{*}\right)\right) & \in \operatorname{Epi}\left(\phi+\delta_{M}\right)^{*}=\operatorname{cl}\left(\operatorname{Epi} \phi^{*}+\{0\} \times Y^{\prime} \times \mathbb{R}_{+}\right) \\
& =\operatorname{Epi} \phi^{*}+\{0\} \times Y^{\prime} \times \mathbb{R}_{+},
\end{aligned}
$$

where we used (3.4) in the inclusion, Lemma 2.1 in the first equality and assumption (ii) in the last one. The above inclusion implies that $\left(-x^{*}, 0,-\alpha\left(x^{*}\right)\right)=(u, v, \gamma)+$ $(0, w, r)$, with $\phi^{*}(u, v) \leq \gamma$ and $r \geq 0$. Therefore,

$$
\alpha\left(x^{*}\right)=-\gamma-r \leq-\gamma \leq-\phi^{*}\left(-x^{*}, v\right) \leq \max _{z \in Y^{\prime}}-\phi^{*}\left(-x^{*}, z\right) \leq \alpha\left(x^{*}\right),
$$

where we used Fenchel-Young inequality in the last inequality. The above expression yields the equality in condition (i) for this choice of $x^{*}$.

It is worth noting that Theorem 3.1 gives a necessary and sufficient condition for the equality

$$
\phi(\cdot, 0)^{*}\left(-x^{*}\right)=\min _{v \in Y^{\prime}} \phi^{*}\left(x^{*}, v\right), \quad \forall x^{*} \in X^{\prime},
$$

which is equivalent to the equality (i) of Theorem 3.1. For numerous sufficient conditions for this equality, see Theorem 2.7.1 and Corollary 2.7.3 of [20].

Corollary 3.1 (Generalized Conjugate Duality) Let $\phi: X \times Y \rightarrow \mathbb{R} \cup\{+\infty\}$ be a proper and lower semi-continuous convex function such that $\alpha:=\inf _{x \in X} \phi(x, 0)<$ $+\infty$. If Epi $\phi^{*}+\left(\{0\} \times Y^{\prime} \times \mathbb{R}_{+}\right)$is weak $k^{*}$ closed then

$$
\inf _{x \in X} \phi(x, 0)=\max _{v \in Y^{\prime}}-\phi^{*}(0, v) .
$$

Proof. The conclusion follows from Theorem 3.1 by taking $x^{*}=0$.

Let us see how the epigraph closure condition in the conjugate duality results can be expressed in the particular case where $\phi$ is described in terms of the sum of two convex functions. Define the continuous linear projection map, $P_{X^{\prime} \times \mathbf{R}}: X^{\prime} \times$ $Y^{\prime} \times \mathbb{R} \rightarrow X^{\prime} \times \mathbb{R}$, by $P_{X^{\prime} \times \mathbf{R}}(u, v, r)=(u, r)$. The inverse of the projection map is denoted by $P_{X^{\prime} \times \mathbf{R}}^{-1}$. Recall that for a continuous linear map $A: X \rightarrow Y$ the adjoint operator of $A$, denoted by $A^{\star}$, is the unique linear application from $Y^{\prime}$ to $X^{\prime}$ with 
the property $\left(A^{\star} v\right)(x)=v(A x)$ for all $v \in Y^{\prime}, x \in X$. Associated to the linear mapping $A$, we consider the mapping $A^{\star} \times I: Y^{\prime} \times \mathbb{R} \rightarrow X^{\prime} \times \mathbb{R}$ defined as $\left(A^{\star} \times\right.$ $I)(w, \alpha)=\left(A^{\star} w, \alpha\right)$. Note that the image of Epi $g^{*}$ through this application is $\left(A^{\star} \times\right.$ $I)\left(\right.$ Epi $\left.g^{*}\right)=\left\{\left(A^{\star} w, \alpha\right) \mid(w, \alpha) \in\right.$ Epi $\left.g^{*}\right\}=\left\{\left(A^{\star} w, \alpha\right) \mid g^{*}(w) \leq \alpha\right\}$. Let $\left(\text { Epi } g^{*}\right)_{A}:=$ $\left(A^{\star} \times I\right)\left(\right.$ Epi $\left.g^{*}\right)$.

Lemma 3.1 Let $X$ and $Y$ be Banach spaces and take $f: X \rightarrow \mathbb{R} \cup\{+\infty\}$ and $g: Y \rightarrow \mathbb{R} \cup\{+\infty\}$ two proper functions. Let $A: X \rightarrow Y$ be a continuous linear mapping. If $\phi: X \times Y \rightarrow \mathbb{R} \cup\{+\infty\}$ is given by $\phi(x, y):=f(x)+g(A x+y)$, then

$$
\text { Epi } \phi^{*}+\left(\{0\} \times Y^{\prime} \times \mathbb{R}_{+}\right)=P_{X^{\prime} \times \mathbf{R}}^{-1}\left(\text { Epi } f^{*}+\left(\text { Epi } g^{*}\right)_{A}\right)
$$

Proof. Call $E:=P_{X^{\prime} \times \mathbf{R}}^{-1}\left(\right.$ Epi $\left.f^{*}+\left(\text { Epi } g^{*}\right)_{A}\right)$ and $F:=$ Epi $\phi^{*}+\left(\{0\} \times Y^{\prime} \times \mathbb{R}_{+}\right)$. Let $(u, v, r) \in E$. Then, $(u, r) \in \operatorname{Epi} f^{*}+\left(\operatorname{Epi} g^{*}\right)_{A}$. So, there exist $\left(u_{1}, r_{1}\right) \in \operatorname{Epi} f^{*}$ and $\left(u_{2}, r_{2}\right) \in$ Epi $g^{*}$ such that

$$
(u, r)=\left(u_{1}+A^{*} u_{2}, r_{1}+r_{2}\right) .
$$

Since $\phi^{*}\left(u_{1}, u_{2}\right)=f^{*}\left(u_{1}-A^{*} u_{2}\right)+g^{*}\left(u_{2}\right), \phi^{*}\left(u_{1}+A^{*} u_{2}, u_{2}\right)=f^{*}\left(u_{1}\right)+g^{*}\left(u_{2}\right) \leq r_{1}+r_{2}$, and so, $\left(u_{1}+A^{*} u_{2}, u_{2}, r_{1}+r_{2}\right) \in \operatorname{Epi} \phi^{*}$. Hence,

$$
(u, v, r)=\left(u_{1}+A^{*} u_{2}, u_{2}, r_{1}+r_{2}\right)+\left(0, v-u_{2}, 0\right) \in F
$$

thus, $E \subset F$.

Conversely, let $(u, v, r) \in F$. Then, $(u, v, r)=\left(u_{1}, u_{2}, r_{1}\right)+\left(0, v, r_{2}\right)$, where $\phi^{*}\left(u_{1}, u_{2}\right)=$ $f^{*}\left(u_{1}-A^{*} u_{2}\right)+g^{*}\left(u_{2}\right) \leq r_{1}, v \in Y^{\prime}$ and $r_{2} \in \mathbb{R}_{+}$. So, $\left(u_{1}-A^{*} u_{2}, r_{1}-g^{*}\left(u_{2}\right)\right) \in \operatorname{Epi} f^{*}$ and $\left(u_{2}, g^{*}\left(u_{2}\right)+r_{2}\right) \in \operatorname{Epi} g^{*}$. Hence,

$$
(u, r)=\left(u_{1}-A^{*} u_{2}+A^{*} u_{2}, r_{1}-g^{*}\left(u_{2}\right)+g^{*}\left(u_{2}\right)+r_{2}\right) \in\left(\operatorname{Epif}^{*}+\left(\operatorname{Epig}^{*}\right)_{A}\right) ;
$$

thus, $F \subset E$.

Note that if $\phi(x, y):=f(x)+g(x+y)$, then

$$
\text { Epi } \phi^{*}+\left(\{0\} \times Y^{\prime} \times \mathbb{R}_{+}\right)=P_{X^{\prime} \times \mathbf{R}}^{-1}\left(\text { Epi } f^{*}+\operatorname{Epi} g^{*}\right) .
$$

Remark 3.1 Let $C \subset X^{\prime} \times \mathbb{R}$ be an arbitrary set. Then it is easy to check that the set $P_{X^{\prime} \times \mathbf{R}}^{-1}(C)$ can be identified with $C \times Y^{\prime}$. Therefore, $C$ is weak $k^{*}$ closed if and only if $P_{X^{\prime} \times \mathbf{R}}^{-1}(C)$ is weak ${ }^{*}$ closed.

Theorem 3.2 (Stable Fenchel-Rockafellar Duality) Let $A: X \rightarrow Y$ be a continuous linear mapping. Let $f: X \rightarrow \mathbb{R} \cup\{+\infty\}$ and $g: Y \rightarrow \mathbb{R} \cup\{+\infty\}$ be proper and lower semi-continuous convex functions such that $A(\operatorname{dom} f) \cap \operatorname{dom} g \neq \emptyset$. Then the following statements are equivalent:

(i) $\inf _{x \in X}\left\{f(x)+g(A x)+x^{*}(x)\right\}=\max _{v \in X^{\prime}}\left\{-f^{*}\left(A^{*} v-x^{*}\right)-g^{*}(-v)\right\}, \forall x^{*} \in$ $X^{\prime}$.

(ii) Epi $f^{*}+\left(\operatorname{Epi} g^{*}\right)_{A}$ is weak* closed. 
Proof. Define $\phi: X \times Y \rightarrow \mathbb{R} \cup\{+\infty\}$ by $\phi(x, y):=f(x)+g(A x+y)$ for each $(x, y) \in X \times Y$. Then, $\phi^{*}(u, v)=f^{*}\left(u-A^{\star} v\right)+g^{*}(v)$. So that condition (i) becomes

$$
\inf _{x \in X} \phi(x, 0)+x^{*}(x)=\max _{v \in Y^{\prime}}\left\{-\phi^{*}\left(-x^{*},-v\right)\right\} \forall x^{*} \in X^{\prime} .
$$

Since $A(\operatorname{dom} f) \cap \operatorname{dom} g \neq \emptyset$, there exists $y_{0} \in \operatorname{dom} g$ such that $y_{0}=A x_{0}$ with $x_{0} \in$ $\operatorname{dom} f$. Hence, $\inf _{x \in X} \phi(x, 0) \leq f\left(x_{0}\right)+g\left(A x_{0}\right)<+\infty$. So we can apply Theorem 3.1, and conclude that (3.5) is equivalent to the weak ${ }^{*}$ closedness of Epi $\phi^{*}+\{0\} \times Y^{\prime} \times \mathbb{R}_{+}$. Now the result follows from Lemma 3.1 and Remark 3.1.

Observe that, in the case where $X=Y$ and $A=I$, Theorem 3.2 shows that Epi $f^{*}+$ Epi $g^{*}$ is weak ${ }^{*}$ closed if and only if

$$
(f+g)^{*}\left(x^{*}\right)=\min \left\{f^{*}(u)+g^{*}(v) \mid u+v=x^{*}\right\}, \quad \forall x^{*} \in X^{\prime},
$$

whenever $\operatorname{dom} f \cap \operatorname{dom} g \neq \emptyset$. For related results, see $[5,12,19]$.

Corollary 3.2 (Stable Duality for Convex Programs) Let $A: X \rightarrow \mathrm{Y}$ be a continuous linear mapping, $f: X \rightarrow \mathbb{R} \cup\{+\infty\}$ be a proper and lower semicontinuous convex function. Let $C \subset \operatorname{dom} f$ be a closed convex set, $b \in \mathrm{Y}$ and let $K \subset \mathrm{Y}$ be a closed convex cone. Assume that $A(\mathrm{C}) \cap(K+b) \neq \emptyset$. Then the following statements are equivalent.

(i) $\inf _{\substack{x \in C \\ A x-b \in K}}\left\{f(x)+x^{*}(x)\right\}=\max _{v \in K^{0}}-\left(f+\delta_{C}\right)^{*}\left(A^{*} v-x^{*}\right)+v(b), \forall x^{*} \in X^{\prime}$

(ii) $\operatorname{Epi}\left(f+\delta_{C}\right)^{*}+\left(\operatorname{Epi} \delta_{b+K}^{*}\right)_{A}$ is weak $k^{*}$ closed,

where $K^{0}=\left\{v \in Y^{\prime} \mid v(y) \leq 0, \forall y \in K\right\}$ is the polar cone of $K$.

Proof. By Theorem 3.2, applied to the functions $f+\delta_{C}$ and $g:=\delta_{b+K}$, we readily see the equivalence between (i) and (ii).

Corollary 3.3 (Generalized Fenchel-Rockafellar Duality) Let $A: X \rightarrow Y$ be a continuous linear mapping. Let $f: X \rightarrow \mathbb{R} \cup\{+\infty\}$ and $g: Y \rightarrow \mathbb{R} \cup\{+\infty\}$ be proper and lower semi-continuous convex functions such that $A(\operatorname{dom} f) \cap \operatorname{dom} g \neq \emptyset$. If the set Epi $f^{*}+\left(\operatorname{Epi} g^{*}\right)_{A}$ is weak $k^{*}$ closed, then

$$
\inf _{x \in X}\{f(x)+g(A x)\}=\max _{v \in Y^{\prime}}\left\{-f^{*}\left(A^{\star} v\right)-g^{*}(-v)\right\} .
$$

Proof. The conclusion follows from Theorem 3.2 by taking $x^{*}=0$.

When the functions $f$ and $g$ are sublinear, we have that stable Fenchel duality is equivalent to the subdifferential sum formula.

Corollary 3.4 (Subdifferential Sum Formula)Let $f, g: X \rightarrow \mathbb{R} \cup\{+\infty\}$ be proper lower semi-continuous sublinear functions with $\operatorname{dom} f \cap \operatorname{dom} g \neq \emptyset$. Then the following statements are equivalent:

(i) $\inf _{x \in X}\left\{f(x)+g(x)+x^{*}(x)\right\}=\max _{v \in X^{\prime}}\left\{-f^{*}\left(v-x^{*}\right)-g^{*}(-v)\right\}, \forall x^{*} \in X^{\prime}$.

(ii) $\partial(f+g)(x)=\partial f(x)+\partial g(x), \forall x \in \operatorname{dom} f \cap$ dom $g$. 
(iii) Epi $f^{*}+$ Epi $g^{*}$ is weak* closed.

Proof. [(i) $\Longrightarrow$ (ii)]. This implication is well known and holds for arbitrary proper convex and lower semicontinuous functions, see for instance Theorem 2.1 of [13]. $[($ ii $) \Longrightarrow$ (iii)]. If (ii) holds then $\partial(f+g)(0)=\partial f(0)+\partial g(0)$. Since $f$ and $g$ are proper lower semi-continuous sublinear functions, $\partial(f+g)(0)=\operatorname{cl}(\partial f(0)+\partial g(0))$. So, $\operatorname{cl}(\partial f(0)+\partial g(0))=\partial f(0)+\partial g(0)$; thus, $\partial f(0)+\partial g(0)$ is weak*closed. Hence,

$$
\text { Epi } f^{*}+\operatorname{Epi} g^{*}=\partial f(0) \times \mathbb{R}_{+}+\partial g(0) \times \mathbb{R}_{+}=(\partial f(0)+\partial g(0)) \times \mathbb{R}_{+}
$$

is weak*closed. $[($ iii $) \Longrightarrow(\mathrm{i})]$. This follows from Theorem 3.2, where $X=Y$ and $A=I$.

Corollary 3.5 (Subdifferential Composition Formula) Let $f: X \rightarrow \mathbb{R} \cup$ $\{+\infty\}$ and $g: Y \rightarrow \mathbb{R} \cup\{+\infty\}$ be proper and lower semi-continuous convex functions such that $A(\operatorname{dom} f) \cap \operatorname{dom} g \neq \emptyset$. Let $A: X \rightarrow Y$ be a continuous linear mapping. If the set Epi $f^{*}+\left(\operatorname{Epi~} g^{*}\right)_{A}$ is weak $k^{*}$ closed then for each $x_{0} \in X$,

$$
\partial(f+(g \circ A))\left(x_{0}\right)=\partial f\left(x_{0}\right)+A^{*} \partial g\left(A x_{0}\right) .
$$

Proof. Let $v \in \partial(f+(g \circ A))\left(x_{0}\right)$. Then, for each $x \in X,(f+g \circ A)(x)-(f+(g \circ$ $A))\left(x_{0}\right) \geq v\left(x-x_{0}\right)$, and so, it follows from Corollary 3.3 that there exits $y_{0} \in Y^{\prime}$ such that

$$
\begin{aligned}
(f-v)\left(x_{0}\right)+(g \circ A)\left(x_{0}\right) & =\inf \{(f-v)(x)+g(A x) \mid x \in X\} \\
& =-(f-v)^{*}\left(A^{*} y_{0}\right)-g^{*}\left(-y_{0}\right) .
\end{aligned}
$$

Now, it is easy to show that $A^{*} y_{0} \in \partial(f-v)\left(x_{0}\right)=\partial f\left(x_{0}\right)-\{v\}$, and $-y_{0} \in$ $\partial g\left(A x_{0}\right)$. Indeed, the above equality yields

$$
\begin{aligned}
-(f-v)\left(x_{0}\right)-(g \circ A)\left(x_{0}\right) & =(f-v)^{*}\left(A^{*} y_{0}\right)+g^{*}\left(-y_{0}\right) \\
& \geq\left[\left(A^{*} y_{0}\right)(x)-(f-v)(x)\right]+\left[\left(-y_{0}\right)(y)-g(y)\right]
\end{aligned}
$$

For all $x \in X$ and $y \in Y$. Taking $y=A x_{0}$, we see that $A^{*} y_{0} \in \partial(f-v)\left(x_{0}\right)=\partial f\left(x_{0}\right)-$ $\{v\}$, and choosing $x=x_{0}$, we obtain $-y_{0} \in \partial g\left(A x_{0}\right)$. Thus, $v \in \partial f\left(x_{0}\right)+A^{*} \partial g\left(A x_{0}\right)$. Hence, $\partial(f+(g \circ A))\left(x_{0}\right) \subset \partial f\left(x_{0}\right)+A^{*} \partial g\left(A x_{0}\right)$. The required equality now follows as the reverse inclusion easily holds.

We now show how a stable minimax theorem can be derived from the stable Fenchel-Rockafellar conjugate duality theorem. For related general stable minimax theorems see $[10,11]$ and other references therein.

Corollary 3.6 (Stable Minimax Theorem) Let $A: X \rightarrow Y$ be a continuous linear mapping. Let $C$ and $D$ be closed convex subsets of $X$ and $Y^{\prime}$ respectively. Suppose that $A(C) \cap \operatorname{dom} \sigma_{D} \neq \emptyset$. Then the set Epi $\sigma_{C}+A^{*}(D) \times \mathbb{R}_{+}$is weak closed if and only if

$$
\inf _{x \in C} \sup _{v \in D} v(A x)+x^{*}(x)=\max _{v \in D} \inf _{x \in C} v(A x)+x^{*}(x), \quad \forall x^{*} \in X^{\prime} .
$$


Proof. Let $f=\delta_{C}$ and $g=\delta_{D}^{*}=\sigma_{D}$ in Theorem 3.2. Then $A(\operatorname{dom} f) \cap \operatorname{dom} g=$ $A(C) \cap \operatorname{dom} \sigma_{D} \neq \emptyset$, and it is easy to check that $\left(\operatorname{Epi} \delta_{D}\right)_{A}=A^{\star}(D) \times \mathbb{R}_{+}$. Now, it follows from Theorem 3.2 that Epi $\sigma_{C}+A^{*}(D) \times \mathbb{R}_{+}$is weak* closed if and only if

$$
\inf _{x \in X}\left\{\delta_{C}(x)+\sigma_{D}(A x)+x^{*}(x)\right\}=\max _{u \in Y^{\prime}}\left\{-\sigma_{C}\left(A^{\star} u-x^{*}\right)-\delta_{D}(-u)\right\},
$$

which is equivalent to the equalities

$$
\begin{aligned}
\inf _{x \in C} \sup _{v \in D} v(A x)+x^{*}(x) & =\max _{-u \in D}\left\{-\sigma_{C}\left(A^{\star} u-x^{*}\right)\right\} \\
& =\max _{-u \in D} \inf _{x \in C}\left[x^{*}(x)+\left(A^{\star}(-u)\right)(x)\right] \\
& =\max _{v \in D} \inf _{x \in C}\left(x^{*}+A^{\star} v\right)(x) \\
& =\max _{v \in D} \inf _{x \in C} v(A x)+x^{*}(x) .
\end{aligned}
$$

\section{Duality and Polyhedral Convex Functions}

This section studies the case in which one of the convex functions is a polyhedral convex function, i.e., a function which has as epigraph a polyhedral convex set. Recall that, for $f, g: \mathbb{R}^{n} \rightarrow \mathbb{R} \cup\{+\infty\}$ convex and lower-semicontinuous functions, the classical Fenchel's Duality Theorem [17, Theorem 31.1] states that one has

$$
\inf _{x \in X}\{f(x)+g(x)\}=\max _{v \in X^{\prime}}\left\{-f^{*}(v)-g^{*}(-v)\right\},
$$

when $\operatorname{ri}(\operatorname{dom} f) \cap \operatorname{ri}(\operatorname{dom} g) \neq \emptyset$. However, if one of these functions, say $g$, is polyhedral, the latter condition can be weakened to $\operatorname{ri}(\operatorname{dom} f) \cap \operatorname{dom} g \neq \emptyset$. Moreover, when both functions are polyhedral, then the relative interiors can be replaced by the domains of the functions. This fundamental result has been recently extended to arbitrary Banach spaces in [16, Theorem 3.3], where the concept of relative interior has been replaced by the strong quasi relative interior (see $[14,15,20]$ ), which is denoted by $\operatorname{sqri}(C)$, and defined as

$$
\text { sqri }(C)=\{x \in C \mid \operatorname{cone}(C-x) \text { is a closed subspace }\} \text {. }
$$

Note that if the set $C$ is contained in a finite dimensional space, then $\operatorname{ri}(C)=$ $\operatorname{sqri}(C)$.

In the case in which both functions $f$ and $g$ are lower semi-continuous polyhedral convex functions on $\mathbb{R}^{n}$, then it is well known that both sets Epi $f^{*}$ and Epi $g^{*}$ are closed polyhedrons, and their sum Epi $f^{*}+$ Epi $g^{*}$, being also a polyhedral, is also closed.

We now show that, when $g$ is polyhedral, our closure condition holds whenever $\operatorname{sqri}(\operatorname{dom} f) \cap \operatorname{dom} g \neq \emptyset$. This condition is used in [16, Theorem 3.3], which is stated below.

Theorem 4.1 Let $f: X \rightarrow \mathbb{R} \cup\{+\infty\}$ and $g: Y \rightarrow \mathbb{R} \cup\{+\infty\}$ be convex proper and lower semicontinuous functions, and let $A: X \rightarrow Y$ be a linear and continuous operator. Suppose that $\operatorname{sqri}(A(\operatorname{dom} f)) \cap \operatorname{dom} g \neq \emptyset$ and that $g$ is polyhedral. Then

$$
\inf _{x \in X}\{f(x)+g(A x)\}=\max _{v \in X^{\prime}}\left\{-f^{*}\left(A^{\star} v\right)-g^{*}(-v)\right\} .
$$


Theorem 4.2 Let $f: X \rightarrow \mathbb{R} \cup\{+\infty\}$ and $g: Y \rightarrow \mathbb{R} \cup\{+\infty\}$ be convex proper and lower semicontinuous functions, and let $A: X \rightarrow Y$ be a linear and continuous operator. Suppose that $g$ is polyhedral and that $\operatorname{dom} g \cap \operatorname{sqri}(A(\operatorname{dom} f)) \neq \emptyset$. Then Epi $f^{*}+\left(\text { Epi } g^{*}\right)_{A}$ is weak $k^{*}$ closed.

Proof. Let $x^{*} \in X^{\prime}$ and define $\hat{f}(x):=f(x)+x^{*}(x)$. Then, $\hat{f}$ is also a proper convex function and $\operatorname{dom} \hat{f}=\operatorname{dom} f$, so that we still have $\operatorname{dom} g \cap \operatorname{sqri}(A(\operatorname{dom} \hat{f})) \neq \emptyset$. Moreover, for each $v \in X^{\prime}, \hat{f}^{*}(v)=f^{*}\left(v-x^{*}\right)$. By Theorem 4.1, we know that if $g$ is polyhedral and dom $g \cap \operatorname{sqri}(A(\operatorname{dom} \hat{f})) \neq \emptyset$, then

$$
\inf _{x \in X}(\hat{f}(x)+g(A x))=\max _{v \in X^{*}}\left(-\hat{f}^{*}\left(A^{\star} v\right)-g^{*}(-v)\right) .
$$

Re-writing the expression above we get

$$
\inf _{x \in X}\left(f(x)+g(A x)+x^{*}(x)\right)=\max _{v \in X^{*}}\left(-f^{*}\left(A^{\star} v-x^{*}\right)-g^{*}(-v)\right) .
$$

Having shown the above equality for arbitrary $x^{*} \in X^{\prime}$, Theorem 3.2 applies and we can conclude that Epi $f^{*}+\left(\text { Epi } g^{*}\right)_{A}$ is weak ${ }^{*}$ closed.

A corollary of the above result is an application to convex programs with polyhedral constraints.

Corollary 4.1 (Duality for Convex Programs with Polyhedral Constraints) Let $A: X \rightarrow Y$ be a continuous linear mapping, $f: X \rightarrow \mathbb{R} \cup\{+\infty\}$ be a proper and lower semi-continuous convex function. Let $C$ be a closed convex subset of $\operatorname{dom} f$, $b \in Y$ and let $K \subset Y$ be a polyhedral cone. Assume that $A(C) \cap(K+b) \neq \emptyset$. Under one of the following conditions:

(i) $Y=\mathbb{R}^{n}$ and $\operatorname{ri}(A(C)) \cap(b+K) \neq \emptyset$,

(ii) $\operatorname{sqri}(A(C)) \cap(b+K) \neq \emptyset$,

(iii) $\operatorname{Epi}\left(f+\delta_{C}\right)^{*}+\left(\operatorname{Epi} \delta_{b+K}^{*}\right)_{A}$ is weak ${ }^{*}$ closed,

one has

$$
\inf \{f(x) \mid x \in C, A x-b \in K\}=\max \left\{-\left(f+\delta_{C}\right)^{*}\left(A^{*} v\right)+v(b) \mid v \in K^{0}\right\} .
$$

Proof. By Corollary 3.2, we have that condition (iii) above implies (4.9), which is condition (i) of Corollary 3.2 for the choice $x^{*}=0$. Using now Theorem 4.2 applied to the functions $f+\delta_{C}$ and $g:=\delta_{b+K}$, we have that both (ii) and its finite dimensional version (i) are stronger than (iii).

\section{References}

[1] H. Attouch and H. Brézis, Duality for the sum of convex functions in general Banach spaces, in Aspects of Mathematics and its applications, J. A. Barroso (ed.), North Holland, Amsterdam, (1986), 125-133. 
[2] H. H. Bauschke, J. M. Borwein and W. Li, Strong conical hull intersection property, bounded linear regularity, Jameson's property $(\mathrm{G})$, and error bounds in convex optimization, Math. Progr., 86 (1999), 135-160.

[3] J. M. Borwein and A. Lewis, Partially finite convex programming, Part I: Quasirelative interiors and duality theory, Math. Progr., 57 (1992), 15 - 48.

[4] R. S. Burachik and V. Jeyakumar, A simple closure condition for the normal cone intersection formula, Applied Mathematics Research Report AMR04, University of New South Wales, Sydney, Australia. To appear in Proc. Amer. Math. Soc. (2005).

[5] R. S. Burachik and V. Jeyakumar, A new geometric condition for Fenchel's duality in infinite dimensions, To appear in Math. Progr., Series B, 2005.

[6] R. S. Burachik and V. Jeyakumar, A dual condition for the convex subdifferential sum formula with applications. To appear in J. Convex Analysis, 12(2) (2005).

[7] F. Deutsch, Best approximation in inner product spaces, Springer-Verlag, New York, 2000.

[8] F. Deutsch, W. Li and J. Swetits, Fenchel duality and the strong conical hull intersection property, J. Optim. Theory Appl., 102 (1999), 681-695.

[9] F. Deutsch, W. Li and J. D. Ward, Best approximation from the intersection of a closed convex set and a polyhedron in Hilbert space, weak Slater conditions, and the strong conical hull intersection property, SIAM J. Optim., 10 (1999), $252-268$.

[10] J. Gwinner and V. Jeyakumar, Stable minimax on noncompact sets. Fixed point theory and applications (Marseille, 1989), Pitman Res. Notes Math. Ser., 252, Longman Sci. Tech., Harlow, 215-220, 1991.

[11] J. Gwinner and J-C. Pomerol, On weak* closedness, coerciveness, and inf-sup theorems, Arch. Math., 52(2) (1989), 159-167.

[12] J.-B. Hiriart-Urruty, $\epsilon$-subdifferential calculus, in Convex Analysis and Optimization, Pitman, Boston, 43-92, 1982.

[13] J.-B. Hiriart-Urruty and R. R. Phelps, Subdifferential Calculus using $\varepsilon-$ subdifferentials, J. of Funct. Anal., 118 (1993), 154-166.

[14] V. Jeyakumar, Duality and infinite dimensional optimization, Nonlinear Anal., 15 (1990), 1111-1122.

[15] V. Jeyakumar and H. Wolkowicz, Generalizations of Slater 's constraint qualification for infinite convex programs, Math. Progr., 57(1) (1992), 85-102.

[16] K. F. Ng and W. Song, Fenchel duality in infinite-dimensional setting and its applications, Nonlinear Analysis 55(7-8) (2003), 845-858. 
[17] R. T. Rockafellar, Convex Analysis, Princeton University Press, Princeton, NJ, 1970 .

[18] T. Strömberg, The operation of infimal convolution, Diss. Math., 352 (1996), $1-61$.

[19] C. Zalinescu, Stability for a class of nonlinear optimization problems and applications, in Nonsmooth Optimization and Related Topics, F. H. Clarke, V. F. Demyanov and F. Giannessi (eds.), Plenum Press, New York, 1989, 437-458.

[20] C. Zalinescu, Convex analysis in general vector spaces, World Scientific, London, 2002. 